\title{
Development of an assessment sheet for fall prediction in stroke inpatients in convalescent rehabilitation wards in Japan
}

\author{
Youichi Nakagawa Katsuhiko Sannomiya $\cdot$ Makiko Kinoshita $\cdot$ Tsutomu Shiomi \\ Kouhei Okada · Hisayo Yokoyama · Yukiko Sawaguti · Keiko Minamoto $\cdot$ Chang-nian Wei \\ Shoko Ohmori · Susumu Watanabe $\cdot$ Koichi Harada $\cdot$ Atsushi Ueda
}

Received: 19 March 2007 / Accepted: 10 December 2007/Published online: 5 April 2008

(C) The Japanese Society for Hygiene 2008

\begin{abstract}
Objective We conducted a study to develop an assessment sheet for fall prediction in stroke inpatients that is handy and reliable to help ward staff to devise a fall prevention strategy for each inpatient immediately upon admission.

Methods The study consisted of three steps: (1) developing a data sampling form to record variables related to risk of falls in stroke inpatients and conducting a follow-up
\end{abstract}

Y. Nakagawa $(\bowtie) \cdot$ K. Minamoto · C. Wei .

S. Ohmori · A. Ueda

Department of Preventive and Environmental Medicine,

Graduate School of Medical and Pharmaceutical Sciences,

Kumamoto University, 1-1-1 Honjo,

Kumamoto 860-8556, Japan

e-mail: youhey@fc.kuh.kumamoto-u.ac.jp

K. Sannomiya $\cdot$ S. Watanabe

Kumamoto Kinoh Hospital, Kumamoto, Japan

M. Kinoshita

Hatsudai Rehabilitation Hospital, Tokyo, Japan

T. Shiomi

Morinomiya Hospital, Osaka, Japan

K. Okada

Chikamori Rehabilitation Hospital, Kochi, Japan

H. Yokoyama

Ukai Rehabilitation Hospital, Nagoya, Japan

Y. Sawaguti

Ohta Atami Hospital, Fukushima, Japan

K. Harada

Department of Microbiology and Environmental

Chemistry, School of Health Sciences,

Kumamoto University, Kumamoto, Japan survey for stroke inpatients from their admission to discharge by using the form; (2) carrying out analyses of characteristics of the present subjects and selecting variables showing a high hazard ratio (HR) for falls using the Cox regression analysis; (3) developing an assessment sheet for fall prediction involving variables giving the integral coefficient for each variable in accordance with the HR determined in the second step.

Results and discussion (1) Subjects of the present survey were 704 inpatients from 17 hospitals including 270 fallers. (2) We selected seven variables as predictors of the first fall: central paralysis, history of previous falls, use of psychotropic medicines, visual impairment, urinary incontinence, mode of locomotion and cognitive impairment. (3) We made 960 trial models in combination with possible coefficients for each variable, and among them we finally selected the most suitable model giving coefficient number 1 to each variable except mode of locomotion, which was given 1 or 2 . The area under the ROC curve of the selected model was 0.73 , and sensitivity and specificity were 0.70 and 0.69 , respectively ( $4 / 5$ at the cut-off point). Scores calculated from the assessment sheets of the present subjects by adding coefficients of each variable showed normal distribution and a significantly higher mean score in fallers $(4.94 \pm 1.29)$ than in non-fallers $(3.65 \pm 1.58)$ $(P=0.001)$. The value of the Barthel Index as the index of ADL of each subject was indicated to be in proportion to the assessment score of each subject.

Conclusion We developed an assessment sheet for fall prediction in stroke inpatients that was shown to be available and valid to screen inpatients with risk of falls immediately upon admission.

Keywords Fall - Stroke - Risk factor - Rehabilitation · Assessment 


\section{Introduction}

Stroke patients are encouraged to start rehabilitation in the early stage from the onset of stroke because early mobilization and more aggressive rehabilitation are recognized to improve functional outcomes of the patients [1-3]. From this view point, the application of an effective stroke management system in combination with rehabilitative treatments from the acute phase of stroke is important [3-6].

Usually in Japan, stroke patients undergo rehabilitation in three stages: acute, convalescent and maintenance. It is necessary to build a systematized cooperation between hospitals for those three stages so that stroke inpatients can receive the appropriate rehabilitation smoothly at each stage [7].

The purpose of convalescent rehabilitation is to regain mobility and independence in activity of daily life (ADL) and to prevent muscle weakness. It has been proven that aggressive rehabilitation from admission to convalescent rehabilitation wards after receiving treatment during the acute period may rapidly improve the ADL of stroke patients [8]. However, inpatients are not accustomed to the ward environment for a while after admission and that situation may increase the chance of falls in the early stage of rehabilitation [9, 10]. In addition, stroke inpatients often have cognitive impairment and high cortical dysfunction, increasing the risk of falls further [11-14].

Falls may have serious consequences for patients receiving rehabilitation, such as injury and disability [15]. Moreover, the psychological changes such as fear of falls may result in self-induced restriction in each activity [16, 17]. Those consequences have a negative effect on the rehabilitation process and its outcome and may result in extended hospitalization and increased medical expense [17].

The risk factors for falls are based on multiple factors such as the physical faculty, neuropsychological and environmental situations of inpatients. It is necessary to consider external and internal factors inclusively to develop fall prevention strategies for inpatients undergoing rehabilitation [18]. On the other hand, unnecessary restriction of activity to prevent falls goes against the purpose of rehabilitation. Therefore, the staff in the rehabilitation wards faces a dilemma balancing aggressive rehabilitation and fall prevention.

In convalescent rehabilitation wards the staff of various occupational categories cooperates to support rehabilitation of stroke inpatients, and all of them commonly recognize the risk of falls among inpatients $[18,19]$. It is also recognized that falls frequently occur within the first week after starting rehabilitation [20].
To date, there have been many reports on the method of assessment for fall prediction among inpatients in rehabilitation wards [18]. However, there are hardly any reports on the method focusing on stroke inpatients who are the majority of patients in convalescent rehabilitation wards in Japan. These facts indicate that it is necessary for the staff in convalescent rehabilitation wards to provide a handy and reliable method of screening inpatients who will be prone to falls immediately upon admission.

From the above viewpoint we developed a form of assessment sheet for fall prediction in stroke patients so that ward staff can use it to devise a fall prevention strategy immediately upon admission of each inpatient by using available and appropriate data that the staff can easily obtain in their work place.

\section{Methods}

We developed an assessment sheet for fall prediction for stroke inpatients by three steps as follows.

Data sampling for selecting variables as an index for an assessment sheet for fall prediction in stroke inpatients

Development of a data sampling form for recording factors related to falls of inpatients in convalescent rehabilitation wards

We organized a workshop team with ten members consisting of medical and co-medical staff engaged in convalescent rehabilitation wards and research staff of the departments of epidemiology and social medicine in Kumamoto University to develop an assessment sheet for stroke inpatients in convalescent rehabilitation wards. Firstly, we established the definition of fall and diagnosis of stroke. A fall was defined as follows: when part of the body above the knee comes in contact with the floor surface against the patient's will [19]. The diagnosis of stroke was based on brain CT, MRI and clinical examination by neurologists.

Next, we collected factors related to falls on the basis of expert knowledge of the workshop members and on the basis of articles on falls associated with rehabilitation. Among factors collected we selected items as independent variables of the present sampling form to be associated with falls of stroke inpatients in convalescent rehabilitation wards and to be easy to use for the staff of wards immediately upon admission of each patient.

The factors chosen as independent variables for falls were as follows: age, sex, presence or absence of central paralysis, history of previous falls from the day of stroke onset to the day before admission to the convalescent 
rehabilitation ward (history of previous falls), use of psychotropic medicines, consciousness disturbance, delirium, depression, visual impairment, sensory disturbance, ataxia, high cortical dysfunctions (e.g., apraxia, aphasia, unilateral spatial neglect and attention disturbance), urinary incontinence, fecal incontinence, the mode of locomotion (walk independently, walk with a cane, walk with a walker, use a wheelchair and on a stretcher), pain, cognitive impairment and ADL.

As for determination of cognitive impairment, we adopted the Revised Hasegawa's Dementia Scale (HDS$\mathrm{R})$. The HDS-R measures the level of cognitive impairment of not only ordinary patients, but also disabled patients with motor and visual impairment [21, 22]. As for determination of ADL, we adopted the Barthel Index (BI). BI measures personal levels of functional independence in ADL [23, 24].

Using the above factors we developed a questionnaire for data sampling of falls in convalescent rehabilitation wards. We developed three types of questionnaire for each patient for use upon admission, at every time a fall occurred and upon discharge.

\section{Method of questionnaire survey}

There were 17 convalescent rehabilitation wards participating in the present survey. They were all members of the conference of convalescent rehabilitation wards in Japan. The structures, procedure of rehabilitation and organization of staff were similar among these wards.

The present sampling survey was approved by the ethics committee of each facility. All of the inpatients in the 17 wards, after receiving acute treatment for cerebrovascular attack, were registered as the subjects of the present study after obtaining written informed consent. If the inpatients did not have the ability to consent, we got the consent from their family. The subjects were followed up from their admission to discharge. The follow-up period per inpatient was less than 3 months. The study period was from 1 June 2004 to 31 June 2005.

The occurrence of falls was reported by the staff, inpatients or inpatients' families, and all data of the subjects were confirmed and recorded by the staff of each facility following the format that we sent. After finishing the follow-up, the recorded formats were sent to the database settled on by the working group from each ward.

Methods of analyses for selecting variables related to falls

First we conducted simple and cross tabulations of the sampling data. For determination of significance in differences between fallers and non-fallers, $\chi^{2}$-test, $t$ test,
Mann-Whitney $U$ test and Wilcoxon sign rank test were used.

After cross tabulation we performed the univariate Cox regression analysis to select preliminary variables to involve in the assessment sheet for fall prediction using the items showing significant differences between fallers and non-fallers.

Next, using variables selected by the univariate analysis indicating $P<0.10$ as the significant value of the hazard ratio (HR) to falls, we performed the multivariate Cox regression analysis to select variables as appropriate items to include in the assessment sheet. The variables finally selected were indicated to be $P<0.10$ in the significant value of the $\mathrm{HR}$.

Development of assessment sheet for fall prediction

Using those variables selected by the statistical procedure as mentioned above, we developed the assessment sheet for fall prediction according to the procedure as follows.

Firstly, we chose appropriate integral numbers for each variable as a possible coefficient in accordance with the value of HR and $95 \%$ CI by the multivariate Cox regression analysis (see Table 2), if characteristics of the significant variables were present for each subject. If those were absent for each subject, the number given to it was 0 . Secondly, we made trial models of the assessment sheet in combination with all of the numbers given to each variable as a possible coefficient. The total score of each assessment sheet was calculated by adding the coefficient of each variable and used as the score of fall risk of each subject. Thirdly, we performed the receiver operating characteristics (ROC) analysis and determined the area under the ROC curve (AUC) to all of the trial models. Finally, among those models one model indicating the highest value of AUC with appropriate balance of sensitivity and specificity was selected as the most suitable assessment sheet.

Statistical analysis

All data were analyzed by SPSS ver.11 statistical software programs.

\section{Results}

Characteristics of the subjects

A total of 704 stroke patients were admitted to convalescent rehabilitation wards, and all of them were registered as the subjects of the present study with the prescribed onset form. Among them, 270 (38\% of total subjects) were fallers. The range of fall rates in each hospital was $35-40 \%$. 
Of 270 fallers, $37 \%$ had their first fall within 10 days after their admission, and also $60 \%$ of them fell within 4 weeks and $46 \%$ of them experienced two or more falls, i.e., recurrent fallers.

The characteristics of the subjects as fallers and nonfallers are shown in Table 1. The items indicating significantly higher rates in fallers than in non-fallers were as follows: age $(P=0.002)$, history of previous falls $(P<0.001)$, side of hemiparesis $(P<0.001)$, ten clinical signs $(P<0.001 \approx P=0.013)$, use of psychotropic medicines $(P=0.004)$, mode of locomotion $(P<0.001)$, median $(\mathrm{Me})$ of HDS-R $(P<0.001)$, days after onset $(P=0.020)$ and days of hospitalization $(P<0.001)$.

Statistics of BI were as follows (not shown in the table). The median (Me) BI of the subjects was 55 upon admission and 80 upon discharge, showing a significant difference between admission and discharge $(P<0.001)$. Non-fallers (60) showed significantly $(P<0.001)$ higher BI than fallers (40) upon admission. In both non-fallers and fallers the BI upon discharge (85 for non-fallers and 70 for fallers) was better than upon admission $(P<0.001)$ between admission and discharge for non-fallers and fallers.

Analyses for selecting the variables related to falls

After cross tabulation we performed univariate and multivariate regression analyses to select variables to involve in the assessment sheet using the items of the follow-up study except showing no significant difference between fallers and non-fallers. The results of the univariate and multivariate Cox regression analyses are shown in Table 2. In the univariate analysis, 14 variables were selected as preliminary predictors showing strong correlation $(P<0.10)$ to falls, such as presence or absence of central paralysis, history of previous falls, use of psychotropic medicines, delirium, visual impairment, sensory disturbance, apraxia, unilateral spatial neglect, attention disturbance, urinary incontinence, fecal incontinence, pain, mode of locomotion and cognitive impairment (the score under 26 in HDS-R).

By the multivariate regression analysis following the univariate analysis, seven variables with a high $\mathrm{HR}$ $(P<0.10)$ were selected as adoptable variables in the present assessment sheet, such as central paralysis (not laterality), history of previous falls, use of psychotropic medicines, visual impairment, urinary incontinence, mode of locomotion and cognitive impairment (score under 26 in HDS-R).

Development of the assessment sheet for fall prediction for stroke inpatients

The range of given integral numbers as preliminary coefficient of each variable transferred values in accordance with $\mathrm{HR}$ and $95 \%$ CI by the multivariate Cox regression analysis was as follows; 1, 2, 3 and 4 for central paralysis, 1 and 2 for history of previous falls, for psychotropic medicines, for visual impairment and for urinary incontinence, 1, 2, 3, 4 and 5 for mode of locomotion, and 1, 2 and 3 for cognitive impairment (score under 26 in HDS-R).

By the combination with all possible coefficients given to seven variables, we developed 960 trial models of the assessment sheet. For all 960 trial models we calculated AUC, sensitivity and specificity. As shown in Table 3, six trial models showed higher AUC value (0.73) with a smooth curve of ROC than other trial models. Among six trial models, we chose model 1 (see Table 3) as the most suitable model because model 1 showed the most reasonable balance of sensitivity and specificity. As shown in the appendix, the final coefficient of each variable was as follows: 1 for central paralysis, for history of previous fall, use of psychotoropic medicines, visual impairment, urinary incontinence, score under 26 in HDS-R and 1 for walk with walkers or 2 for using a wheel chair as the mode of locomotion. Then the total score of the present assessment sheet was ranged from 0 to 8 . When the cut-off point was set at 4 and 5 , the sensitivity and specificity were indicated to be 0.70 and 0.69 , respectively.

As shown in Table 4, the distribution of the total score calculated from the present assessment sheet applying data of the subjects upon admission was proved to be normal by Kolmogorov-Smirnov test [25] and shows a dose-response relation curve both in all the subjects and in fallers. The mean score of fallers $(4.95 \pm 1.29)$ was higher than that of non-fallers $(3.65 \pm 1.58)(P<0.001)$.

According to the percentile value of the assessment score calculated from each subject, the present subjects were classified into three groups. The subjects with scores of 0 to 2 (0-25th percentile of total subjects) were classified as risk-1, those with scores of 3 to 4 (25-75th percentile) as risk- 2 and those with scores of 5 to 8 (75100 th percentile) as risk-3. The rates of falls were $9.2 \%$ for risk-1, 27.4\% for risk-2 and 58.3\% for risk-3. By KaplanMeier analysis with the log-rank test, a significant difference was found (log rank statistics, 77.98; $P<0.001)$ among three groups as shown in Fig. 1. The Kaplan-Meier curve also showed that at least $85 \%$ of inpatients in risk-1 underwent rehabilitation without experiencing falls during the observation period and, in contrast, $20 \%$ of inpatients in risk-3 experienced a fall within 10 days after admission.

As shown in Table 5, the median BIs upon admission and discharge were 80 and 100 for risk-1, 60 and 80 for risk- 2 and 30 and 60 for isk-3, indicating that the BI of each subject improved depending on the risk group through their admission to discharge. 
Table 1 Characteristics of the subjects in the study

$* \chi^{2}$-test

** Non-faller versus faller: $t$

test

*** Non-faller versus faller:

Mann-Whitney $U$ test

${ }^{a}$ Involving subarachnoid

hemorrhage

b Visual impairment included reduced visual acuity and visual field loss

c Sensory disturbance included anesthesia, hypesthesia,

hyperesthesia, paresthesia and dysesthesia

${ }^{d}$ Pain defined as an unpleasant feeling caused by an actual and underlying damage of the organization

e Psychotropic medicines included antipsychotics, antidepressants, antianxiety drugs, hypnotics and antiepileptics

${ }^{\mathrm{f}}$ Antihypertensives included $\mathrm{Ca}$ antagonists, $\beta$-blockers, angiotensin converting enzyme inhibitors, angiotensin receptor blockers and diuretics

\begin{tabular}{|c|c|c|c|c|c|}
\hline Characteristics & Total & Non-faller & Faller & $P$ & \\
\hline$n(\%)$ & $704(100.0)$ & $434(100.0)$ & $270(100.0)$ & & \\
\hline Sex; n: M/F & $406 / 298$ & $247 / 187$ & $159 / 111$ & NS & $*$ \\
\hline Age; years: mean $\pm S D$ & $69.7 \pm 12.1$ & $68.6 \pm 12.7$ & $71.4 \pm 10.9$ & 0.002 & $* *$ \\
\hline History of previous falls; $n(\%)$ & $85(12.1)$ & $39(9.0)$ & $46(17.0)$ & $<0.001$ & \\
\hline \multicolumn{6}{|l|}{ Diagnoses; $n(\%)$} \\
\hline Cerebral infarction $(I)$ & $403(57.2)$ & $254(58.5)$ & $149(55.2)$ & NS & $*$ \\
\hline Cerebral hemorrhage $(H)^{\mathrm{a}}$ & $241(34.2)$ & $144(33.2)$ & $97(35.9)$ & & \\
\hline$I+H$ & $60(8.5)$ & $36(8.3)$ & $24(8.9)$ & & \\
\hline \multicolumn{6}{|l|}{ Side of hemiparesis [central paralysis]; $n(\%)$} \\
\hline Neither & $76(10.8)$ & $63(14.5)$ & $13(4.8)$ & $<0.001$ & $*$ \\
\hline Right & $250(35.5)$ & $154(35.5)$ & $96(35.6)$ & & \\
\hline Left & $334(47.4)$ & $194(44.7)$ & $140(51.9)$ & & \\
\hline Both & $44(6.3)$ & $23(5.3)$ & $21(7.8)$ & & \\
\hline \multicolumn{6}{|l|}{ Clinical signs; $n(\%)$} \\
\hline Consciousness disturbance & $124(17.6)$ & $64(14.7)$ & $60(22.2)$ & 0.008 & $*$ \\
\hline Delirium & $17(2.4)$ & $6(2.2)$ & $11(4.1)$ & 0.024 & $*$ \\
\hline Depression & $65(9.2)$ & $35(8.1)$ & $30(11.1)$ & NS & $*$ \\
\hline Visual impairment ${ }^{\mathrm{b}}$ & $145(20.6)$ & $74(17.1)$ & $71(26.3)$ & 0.002 & * \\
\hline Sensory disturbance ${ }^{c}$ & $431(61.2)$ & $244(56.2)$ & $187(69.3)$ & $<0.001$ & * \\
\hline Ataxia & $116(16.5)$ & $72(16.6)$ & $44(16.3)$ & NS & $*$ \\
\hline Apraxia & $68(9.7)$ & $33(7.6)$ & $35(13.0)$ & 0.014 & $*$ \\
\hline Aphasia & $106(15.1)$ & $63(14.5)$ & $43(15.9)$ & NS & $*$ \\
\hline Unilateral spatial neglect & $166(23.6)$ & $82(18.9)$ & $84(31.1)$ & $<0.001$ & * \\
\hline Attention disturbance & $322(45.7)$ & $167(38.5)$ & $155(57.4)$ & $<0.001$ & * \\
\hline Urinary incontinence & $282(40.1)$ & $135(31.1)$ & $147(54.4)$ & $<0.001$ & $*$ \\
\hline Fecal incontinence & $173(24.6)$ & $87(20.0)$ & $86(31.9)$ & $<0.001$ & * \\
\hline Pain $^{\mathrm{d}}$ & $182(25.9)$ & $99(22.8)$ & $83(30.7)$ & 0.013 & * \\
\hline Use of psychotropic medicines ${ }^{\mathrm{e}} ; n(\%)$ & $225(32.0)$ & $122(28.1)$ & $103(38.1)$ & 0.004 & $*$ \\
\hline Use of antihypertensives ${ }^{\mathrm{f}} ; n(\%)$ & $305(43.3)$ & $180(41.5)$ & $125(46.3)$ & NS & $*$ \\
\hline \multicolumn{6}{|l|}{ Mode of locomotion; $n(\%)$} \\
\hline Walk independently & $100(14.2)$ & $89(20.5)$ & $11(4.1)$ & $<0.001$ & $*$ \\
\hline Walk with cane & $48(6.8)$ & $40(9.2)$ & $8(3.0)$ & & \\
\hline Walk with walker & $52(7.4)$ & $36(8.3)$ & $16(5.9)$ & & \\
\hline In wheelchair & $490(69.6)$ & $258(59.4)$ & $232(85.9)$ & & \\
\hline On stretcher & $14(2.0)$ & $11(2.5)$ & $3(1.1)$ & & \\
\hline Median HDS-R (1st, 3rd quartiles): 0-30 & $22(14,27)$ & $23(16,28)$ & $20(12,25)$ & $<0.001$ & $* * *$ \\
\hline Onset to admission; day: mean $\pm \mathrm{SD}$ & $40.4 \pm 24.9$ & $38.7 \pm 24.9$ & $43.2 \pm 24.7$ & 0.020 & $* *$ \\
\hline Hospitalization; day: mean \pm SD & $90.8 \pm 48.8$ & $79.0 \pm 46.6$ & $114.2 \pm 44.5$ & $<0.001$ & $* *$ \\
\hline
\end{tabular}

\section{Discussion}

For stroke inpatients in convalescent rehabilitation wards, falls are the most important adverse event to archive a goal of each rehabilitation outcome. For staff of rehabilitation wards receiving such inpatients, they should precisely predict the risk of falls of each inpatient immediately upon admission. However, to date, we have no appropriate method of screening of inpatients who are prone to falls early after admission. From this view point, we developes an assessment sheet for fall prediction of stroke inpatients that can be used effectively by staff of convalescent rehabilitation wards to design a fall prevention strategy immediately upon admission of each inpatient using available data that the staff can easily obtain from each inpatient.

We organized a workshop team on the prevention of falls in those undergoing rehabilitation consisting of medical and co-medical staff in convalescent rehabilitation wards and specialists in preventive medicine of the Kumamoto University staff. 
Table 2 Univariate and multivariate Cox regression analyses on the relationship between characteristics of inpatients upon admission and fall events during hospitalization in convalescent rehabilitation wards $(n=704)$

a The Revised Hasegawa's Dementia Scale

b Hazard ratio

${ }^{c}$ Confidence interval

\begin{tabular}{|c|c|c|c|c|c|c|}
\hline & \multicolumn{3}{|c|}{ Univariate Cox regression } & \multicolumn{3}{|c|}{ Multivariate Cox regression } \\
\hline & $\mathrm{HR}^{\mathrm{b}}$ & $95 \% \mathrm{CI}^{\mathrm{c}}$ & $P$ & $\mathrm{HR}^{\mathrm{b}}$ & $95 \% \mathrm{CI}^{\mathrm{c}}$ & $P$ \\
\hline Sex & 0.84 & $0.66-1.07$ & 0.162 & 0.79 & $0.61-1.01$ & 0.061 \\
\hline Age & 1.01 & $0.98-1.03$ & 0.102 & 1.01 & $0.99-1.02$ & 0.148 \\
\hline History of previous falls & 1.79 & $1.30-2.46$ & $<0.001$ & 1.73 & $1.25-2.40$ & 0.001 \\
\hline Side of hemiparesis (central paralysis) & & & 0.013 & & & 0.062 \\
\hline Neither (reference group) & & & & 1.00 & & \\
\hline Right & 2.18 & $1.24-3.95$ & 0.008 & 2.22 & $1.22-4.02$ & 0.013 \\
\hline Left & 2.43 & $1.38-4.31$ & 0.002 & 2.25 & $1.24-4.08$ & 0.011 \\
\hline Both & 2.88 & $1.54-6.16$ & 0.003 & 2.21 & $1.08-4.52$ & 0.026 \\
\hline \multicolumn{7}{|l|}{ Clinical signs } \\
\hline Consciousness disturbance & 1.22 & $0.91-1.62$ & 0.182 & & & \\
\hline Delirium & 2.48 & $1.36-4.55$ & 0.003 & 1.74 & $0.65-1.26$ & 0.112 \\
\hline Depression & 1.21 & $0.83-1.76$ & 0.334 & & & \\
\hline Visual impairment & 1.40 & $1.07-1.84$ & 0.015 & 1.33 & $0.99-1.80$ & 0.059 \\
\hline Sensory disturbance & 1.34 & $1.04-1.74$ & 0.027 & 1.10 & $0.83-1.47$ & 0.509 \\
\hline Ataxia & 0.97 & $0.70-1.34$ & 0.855 & & & \\
\hline Apraxia & 1.48 & $1.04-2.11$ & 0.030 & 1.32 & $0.89-1.95$ & 0.168 \\
\hline Aphasia & 1.00 & $0.72-1.38$ & 0.977 & & & \\
\hline Unilateral spatial neglect & 1.33 & $1.03-1.73$ & 0.030 & 0.84 & $0.60-1.17$ & 0.304 \\
\hline Attention disturbance & 1.54 & $1.21-1.96$ & 0.001 & 1.11 & $0.82-1.51$ & 0.494 \\
\hline Urinary incontinence & 1.78 & $1.40-2.26$ & 0.001 & 1.58 & $1.12-2.22$ & 0.009 \\
\hline Fecal incontinence & 1.37 & $1.06-1.77$ & 0.016 & 0.73 & $0.51-1.03$ & 0.075 \\
\hline Pain & 1.28 & $0.99-1.66$ & 0.062 & 1.13 & $0.86-1.48$ & 0.392 \\
\hline Use of psychotropic medicines & 1.37 & $1.07-1.76$ & 0.011 & 1.31 & $1.02-1.69$ & 0.032 \\
\hline Use of antihypertensives & 1.19 & $0.93-1.51$ & 0.162 & & & \\
\hline Mode of locomotion & & & 0.001 & & & 0.006 \\
\hline Walk independently (reference group) & & & & 1.00 & & \\
\hline Walk with cane & 1.62 & $0.66-4.05$ & 0.302 & 1.83 & $0.73-4.58$ & 0.199 \\
\hline Walk with walker & 2.77 & $1.29-6.00$ & 0.009 & 2.48 & $1.14-5.38$ & 0.021 \\
\hline In wheelchair & 3.68 & $2.06-6.93$ & 0.001 & 2.96 & $1.60-5.47$ & 0.001 \\
\hline On stretcher & 1.37 & $0.43-5.53$ & 0.627 & 1.03 & $0.29-3.75$ & 0.960 \\
\hline HDS-R ${ }^{a}$ & & & $<0.001$ & & & 0.029 \\
\hline 27-30 (reference group) & & & & 1.00 & & \\
\hline $0-26$ & 2.38 & $1.32-3.34$ & 0.001 & 1.59 & $1.12-2.61$ & 0.042 \\
\hline
\end{tabular}

In the workshop, firstly, we collected items associated with falls in stroke patients undergoing rehabilitation by bibliographic references and technical knowledge of workshop members; among those items collected we selected items that may be obtained easily by any staff of the ward immediately upon admission of each inpatient. Using the items selected we developed case sampling formats on falls for use upon admission, every time a fall event occurred and upon discharge, and using those formats we conducted a follow-up survey for the stroke inpatients in 17 hospitals with similar ward structures, rehabilitation procedures and staff organizations. The procedure of the present study in combination with qualitative research and quantitative and prospective research may confirm the validity of the process of data collecting and of factors related to falls collected from the subjects.

In the follow-up study the present subjects showed a fall rate of $38 \%$, indicating an ordinary fall rate comparable with previous reports of the rehabilitation wards [26, 27]. Among 270 fallers $46 \%$ of them were recurrent fallers. It has been indicated that recurrent fallers might show poor improvement of BI or ADL compared to single and nonfallers $[28,29]$. However, we attached importance to the first fall to be involved in the present assessment sheet because, as our results clarified, many falls occurred within 
Table 3 The trial models of the assessment sheet for fall prediction showing a higher value of $\mathrm{AUC}^{\mathrm{a}}$ a AUC: the area under the ROC curve

\begin{tabular}{|c|c|c|c|c|c|c|}
\hline Variable/coefficient & Model 1 & Model 2 & Model 3 & Model 4 & Model 5 & Model 6 \\
\hline \multicolumn{7}{|l|}{ Central paralysis } \\
\hline Presence & 1 & 2 & 2 & 3 & 3 & 4 \\
\hline Absence & 0 & 0 & 0 & 0 & 0 & 0 \\
\hline \multicolumn{7}{|c|}{ History of previous falls } \\
\hline Presence & 1 & 1 & 2 & 2 & 2 & 2 \\
\hline Absence & 0 & 0 & 0 & 0 & 0 & 0 \\
\hline \multicolumn{7}{|c|}{ Use of psychotropic medicines } \\
\hline Presence & 1 & 1 & 1 & 1 & 2 & 2 \\
\hline Absence & 0 & 0 & 0 & 0 & 0 & 0 \\
\hline \multicolumn{7}{|l|}{ Visual impairment } \\
\hline Presence & 1 & 1 & 1 & 1 & 2 & 2 \\
\hline Absence & 0 & 0 & 0 & 0 & 0 & 0 \\
\hline \multicolumn{7}{|l|}{ Urinary incontinence } \\
\hline Presence & 1 & 1 & 2 & 2 & 2 & 2 \\
\hline Absence & 0 & 0 & 0 & 0 & 0 & 0 \\
\hline \multicolumn{7}{|l|}{ Mode of locomotion } \\
\hline Walk with walker & 1 & 2 & 2 & 3 & 3 & 4 \\
\hline In wheelchair & 2 & 3 & 3 & 4 & 4 & 5 \\
\hline Others & 0 & 0 & 0 & 0 & 0 & 0 \\
\hline \multicolumn{7}{|l|}{ HDS-R } \\
\hline $0-26$ & 1 & 2 & 2 & 2 & 2 & 3 \\
\hline $27-30$ & 0 & 0 & 0 & 0 & 0 & 0 \\
\hline Total & $0-8$ & $0-11$ & $0-13$ & $0-15$ & $0-17$ & $0-20$ \\
\hline AUC & 0.73 & 0.73 & 0.73 & 0.73 & 0.73 & 0.73 \\
\hline Cut off & $4 / 5$ & $7 / 8$ & $7 / 8$ & $9 / 10$ & $9 / 10$ & $11 / 12$ \\
\hline Sensitivity & 0.70 & 0.65 & 0.68 & 0.67 & 0.70 & 0.75 \\
\hline Specificity & 0.69 & 0.72 & 0.68 & 0.69 & 0.66 & 0.58 \\
\hline
\end{tabular}

Table 4 The distribution of subjects by the scores calculated from the assessment sheet

\begin{tabular}{lccc}
\hline Score & $\begin{array}{l}\text { Subjects: } \\
n(\%)^{\mathrm{a}}\end{array}$ & $\begin{array}{l}\text { Cumulative } \\
\text { frequency: } n(\%)^{\mathrm{b}}\end{array}$ & $\begin{array}{l}\text { Fallers: } \\
n(\%)^{\mathrm{c}}\end{array}$ \\
\hline 0 & $5(0.7)$ & $5(0.7)$ & $0(0.0)$ \\
1 & $45(6.4)$ & $50(7.1)$ & $5(11.1)$ \\
2 & $69(9.8)$ & $119(16.9)$ & $6(8.7)$ \\
3 & $109(15.5)$ & $228(32.4)$ & $22(20.2)$ \\
4 & $157(22.3)$ & $385(54.7)$ & $51(32.5)$ \\
5 & $169(24.0)$ & $554(78.7)$ & $95(56.2)$ \\
6 & $120(17.0)$ & $674(95.7)$ & $69(57.5)$ \\
7 & $26(3.7)$ & $700(99.4)$ & $18(69.2)$ \\
8 & $4(0.6)$ & $704(100.0)$ & $4(100.0)$ \\
Total & 704 & & 270 \\
\hline
\end{tabular}

a The number of each score group and percent of the total subjects

b The number of cumulative frequency and percent of the total subjects

c The number of fallers of each score group and percent of each corresponding score group a short time after admission and because the previous report revealed that fallers might develop a psychological change such as fear of falling resulting in self-induced restrictions in activity [16, 17].

The distribution of scores of BI of the present subjects upon discharge showed significant improvement compared to those upon admission for both fallers and non-fallers, indicating that appropriate and effective rehabilitation for each inpatient has been provided in each rehabilitation ward. Nevertheless, BI has been recognized as an important factor related to falls. We excluded BI from the items of the present assessment sheet because BI was usually evaluated on the basis of determination of ADL of the inpatient for about 1 week after admission, and the staff of rehabilitation wards cannot use the data of BI upon admission of each inpatient.

We performed univariate and multivariate Cox regression analysis [30] to select variables to include in the present assessment sheet from the factors adopted in the data-sampling format. The Cox regression analysis is 


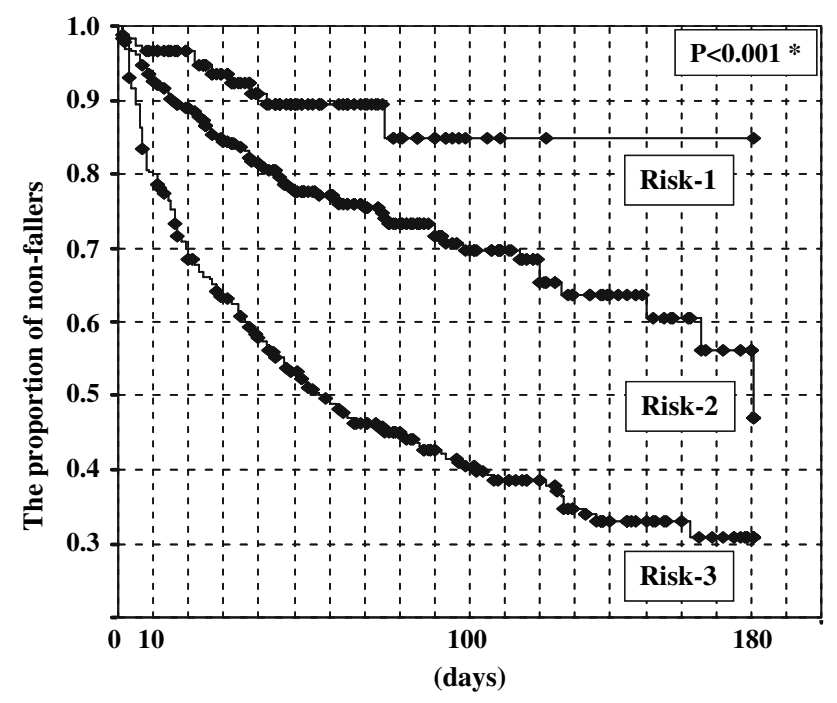

Fig. 1 Kaplan-Meier curves for the risk of falls among three groups. Risk-1: score of 0 to 2, risk-2: score of 3 to 4, risk-3: score of 5 to 8 . *Risk-1 versus risk-2 versus Risk-3: $\log$ rank statistic; $P<0.001$

Table 5 Comparison of Barthel Index among risk groups on the day of admission and discharge: median BI (1st, 3rd quartiles)

\begin{tabular}{|c|c|c|c|c|}
\hline & $n$ & $\begin{array}{l}\text { BI on the day } \\
\text { of admission }\end{array}$ & & $\begin{array}{l}\text { BI on the day } \\
\text { of discharge }\end{array}$ \\
\hline Risk- $1^{a}$ & 101 & $80(60,95)^{*}$ & & $100(80,100) *$ \\
\hline Risk- $2^{a}$ & 453 & $60(40,65)^{*}$ & $* *$ & $80(70,95)^{*}$ \\
\hline Risk- $3^{a}$ & 150 & $30(10,50)^{*}$ & & $60(35,75) *$ \\
\hline
\end{tabular}

${ }^{a}$ Risk 1: score of 0 to 2, risk 2: score of 3 to 4, risk 3: score of 5 to 8

* Admission versus discharge: Wilcoxon sign rank test; $P<0.001$

** Risk-1 versus risk-2 versus risk-3: Mann-Whitney $U$ test; $P<0.001$

the most suitable method to evaluate the intensity of the relationship between falls and each co-variable with common time factors with each other and to determine the value of coefficient of each variable for falls as the HR. Those variables selected by the present statistical methods involve internal factors such as motor, sensory and cognitive ability and external factors such as drug and devices. Findings of other studies on falls among inpatients in rehabilitation wards suggest that such variables selected are definitely valid for fall prediction in stroke inpatients $[15$, $18,19]$. These facts indicate the confirmed validity at each variable selected to be adopted in the present assessment sheet both clinically and statistically.

By the combination of all possible coefficients to seven selected variables based on the $\mathrm{HR}$ and $95 \% \mathrm{CI}$, we developed 960 trial models and chose 6 models with the highest value of AUC. Among those six models we selected model 1 (see Table 3 ) as the most suitable model because it indicates an appropriate balance of sensitivity and specificity.

As shown in the appendix, each of seven variables was given the coefficient number of the presence of each corresponding variable as follows: 1 to central paralysis, history of previous fall, use of psychotoropic medicines, visual impairment, urinary incontinence, score under in HDS-R and 1 or 2 to mode of locomotion. Then, the range of the total score of the present sheet per each subject by adding coefficient numbers of each variable was $0-8$. The distribution of the scores of each subject based on the present sheet was shown to be normal. The mean score of the fallers was significantly higher than that of the nonfallers $(P<0.001)$, and also the dose-response relationship were shown in the scores of both total subjects and that of fallers. Those facts indicate that the present sheet is valid and available for screening stroke inpatients who are prone to falls.

Therefore, we considered an appropriate use of the present assessment sheet. According to the percentile value of each inpatient the subjects were classified into three groups such as risk-1, risk-2 and risk-3. As shown in Fig. 1, a significant difference in fall rate by days after admission was found among three groups by Kaplan-Meier analysis [31]. The Kaplan-Meier curve also showed that more than $20 \%$ of the inpatients in risk-3 experienced a fall within 10 days after admission and, in contrast, the fallers of the risk-1 from admission to discharge were only $15 \%$. The Kaplan-Meier curve indicated that the present assessment model was proved to be effective for identifying high-risk inpatients in the early period after admission of convalescent rehabilitation wards.

As to the BI, it was clarified that the BI of inpatients in each risk group showed a significant decrease in order of risk-1 to 3 both upon admission and upon discharge (Table 5). The result indicates that BI may change depending on the total score of the present sheet and suggests that it is not necessary to adopt $\mathrm{BI}$ in the assessment sheet.

According with these facts we defined risk- 1 to be the low risk group, risk-2 to be the middle risk group and risk-3 to be the high risk group for falls, and we advised the staff of convalescent rehabilitation wards that this classification should be applied to each inpatient by ward staff to devise a fall prevention strategy immediately upon admission.

We are now conducting new follow-up research for the staff of convalescent rehabilitation wards to evaluate the availability and the validity of using the present assessment sheet to screen inpatients who are prone to falls and of conducting the classification of the three risk groups to develop a strategy for the prevention of falls for each inpatient. 


\section{Conclusion}

We developed an assessment sheet consisting of seven variables for fall prediction for stroke inpatients that can be applied to screen inpatients with a fall risk immediately upon admission. The assessment sheet consists of variables that staff of convalescent rehabilitation wards can easily obtain from data of inpatients upon admission. We recommend that the staff of convalescent rehabilitation wards should use the present assessment sheet immediately upon admission of each inpatient and classify each inpatient into three groups according to the assessment score in order to devise a strategy for fall prevention.

Acknowledgements The study was supported by the Medical Care Safety Committee of the Liaison Council concerning the National Convalescent Rehabilitation Care Unit. The authors express many thanks to Dr. Masahiro Shono, Yuge Hospital, Kumamoto Japan, for his valuable statistical advice and help.

\section{Appendix}

Final assessment sheet for prediction of falls with the coefficient score of each variable by presence and absence in each subject

\begin{tabular}{|c|c|c|}
\hline Variable & Score (weight) & \\
\hline \multicolumn{3}{|l|}{ Central paralysis } \\
\hline Presence & 1 & \\
\hline Absence & 0 & \\
\hline \multicolumn{3}{|c|}{ History of previous falls } \\
\hline Presence & 1 & \\
\hline Absence & 0 & \\
\hline \multicolumn{3}{|c|}{ Use of psychotropic medicines } \\
\hline Presence & 1 & \\
\hline Absence & 0 & \\
\hline \multicolumn{3}{|l|}{ Visual impairment } \\
\hline Presence & 1 & \\
\hline Absence & 0 & \\
\hline \multicolumn{3}{|l|}{ Urinary incontinence } \\
\hline Presence & 1 & \\
\hline Absence & 0 & \\
\hline \multicolumn{3}{|l|}{ Mode of locomotion } \\
\hline Walk with walker & 1 & 1 \\
\hline In wheelchair & 2 & \\
\hline Others & 0 & \\
\hline \multicolumn{3}{|l|}{ HDS-R } \\
\hline $0-26$ & 1 & \\
\hline $27-30$ & 0 & \\
\hline Total & $0-8$ & \\
\hline
\end{tabular}

${ }^{1}$ You choose one item from these

\section{References}

1. Chan KM, Wong SF, Yoong T. Early rehabilitation for stroke patients: a new look. Singapore Med J. 1998;39:451-5.

2. Maulden SA, Gassaway J, Horn SD, Smout RJ, Dejong G. Timing of initiation of rehabilitation after stroke. Arch Phys Med Rehabil. 2005;86:34-40.

3. Novack TA, Satterfield WT, Lyons K, Kolski G, Hackmeyer L, Connor M. Stroke onset and rehabilitation: time lag as a factor in treatment outcome. Arch Phys Med Rehabil. 1984;65:316-9.

4. Hamrin E. Early activation in stroke: does it make a difference? Scand J Rehabil Med. 1982;14:101-9.

5. Ishigami S. Rehabilitation approaches to CVA in the acute stage. Nippon Ronen Igakkai Zasshi. 2000;37:892.

6. Paolucci S, Antonucci G, Grasso MG, Morelli D, Troisi E, Coiro $P$, et al. Early versus delayed inpatient stroke rehabilitation: a matched comparison conducted in Italy. Arch Phys Med Rehabil. 2000;81:695-700.

7. Hashimoto Y, Terasaki T, Yonehara T, Tokunaga M, Hirano T, Uchino M. Critical pathway and hospital-hospital cooperation in acute stroke. Reduction of the length of hospital stay. Interventional Neuroradiol. 2000;6:251-5.

8. Suzuki T, Sonoda S, Misawa K, Saitoh E, Shimizu Y, Kotake T. Incidence and consequence of falls in inpatient rehabilitation of stroke patients. Exp Aging Res. 2005;31:457-69.

9. Patel M, Potter J, Perez I, Kalra L. The process of rehabilitation and discharge planning in stroke: a controlled comparison between stroke units. Stroke. 1998;29:2484-7.

10. Shiraishi N, Mizutani C, Menjho M, Deguchi A, Takase K, Hamaguchi $\mathrm{H}$, et al. Comparison of activities of daily living for a convalescent rehabilitation ward and general ward for stroke patients. Nippon Ronen Igakkai Zasshi. 2004;41:646-52.

11. Horikawa E, Matsui T, Arai H, Seki T, Iwasaki K, Sasaki H. Risk of falls in Alzheimer's disease: a prospective study. Intern Med. 2005;44:717-21.

12. LaBuda J, Lichtenberg P. The role of cognition, depression, and awareness of deficit in predicting geriatric rehabilitation patients' IADL performance. Clin Neuropsychol. 1999;13:258-67.

13. Rapport LJ, Webster JS, Flemming KL, Lindberg JW, Godlewski $\mathrm{MC}$, Brees JE, et al. Predictors of falls among right-hemisphere stroke patients in the rehabilitation setting. Arch Phys Med Rehabil. 1993;74:621-6.

14. Van Doorn C, Gruber-Baldini AL, Zimmerman S, Hebel JR, Port CL, Baumgarten M, et al. Epidemiology of Dementia in Nursing Homes Research Group. Dementia as a risk factor for falls and fall injuries among nursing home residents. J Am Geriatric Soc. 2003;51:1213-8.

15. Mayo NE, Korner-Bitensky N, Levy AR. Risk factors for fracture due to fall. Arch Phys Med Rehabil. 1993;74:917-21.

16. Liu-Ambrose T, Khan KM, Eng JJ, Lord SR, McKay HA. Balance confidence improves with resistance or agility training. Increase is not correlated with objective changes in fall risk and physical abilities. Gerontology. 2004;50:373-82.

17. Tinetti ME, Williams CS. Falls, injuries due to falls, and the risk of admission to a nursing home. N Engl J Med. 1997;337: 1279-84.

18. Perell KL, Nelson A, Goldman RL, et al. Fall risk assessment measures: an analytic review. J Gerontol Med Sci. 2001;56A: 761-6.

19. Nyberg L, Gustafson Y. Fall prediction index for patients in stroke rehabilitation. Stroke. 1997;28:716-21.

20. Vassallo M, Sharma JC, Briggs RS, Allen SC. Characteristics of early fallers in elderly patient rehabilitation wards. Age Ageing. $2003 ; 32: 338-42$. 
21. Imai Y, Hsegawa K. The revised Hasegawa's Dementia Scale (HDS-R)-Evaluation of its usefulness as a screening test for dementia. J Hong Kong Coll Psychiatr. 1994;4:20-4.

22. Kim KW, Lee DY, Jhoo JH, Youn JC, Suh YJ, Jun YH, et al. Diagnostic accuracy of mini-mental status examination and revised hasegawa dementia scale for Alzheimer's disease. Dement Geriatr Cogn Disord. 2005;19:324-30.

23. Kwon S, Hartzema AG, Duncan PW, Min-Lai S. Disability measures in stroke: relationship among the Barthel Index, the Functional Independence Measure, and the Modified Rankin Scale. Stroke. 2004;35:918-23.

24. Mahoney FI, Barthel D. Functional evaluation: the Barthel Index. Md Med J. 1965;14:56-61.

25. Armitage P, Berry G. Statistical methods in medical research, 3rd edn. 2001;355-7. (Japanese translation)

26. Nyberg L, Gustafson Y. Patient falls in stroke rehabilitation. A challenge to rehabilitation strategies. Stroke. 1995;26:838-42.
27. Teasell R, McRae M, Foley N, Bhardwaj A. The incidence and consequences of falls in stroke patients during inpatient rehabilitation: factors associated with high risk. Arch Phys Med Rehabil. 2002;83:329-33.

28. Luukinen H, Koski K, Laippala P, Kivela SL. Risk factors for recurrent falls in the elderly in long-term institutional care. Public Health. 1995;109:57-65.

29. Vassallo M, Sharma JC, Allen SC. Characteristics of single fallers and recurrent fallers among hospital in-patients. Gerontology. 2002;48:147-50.

30. Cox DR. Regression models and life tables. J R Stat Soc. 1972;B34:187-220.

31. Kaplan EL, Meier P. Nonparametric estimation from incomplete observations. J Am Stat Assoc. 1958;53:457-81. 